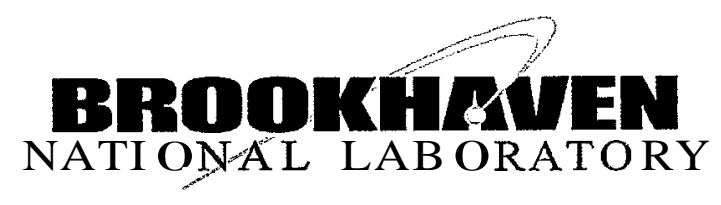

BNL-73478-2005-CP

\title{
Interaction Region Design for the Electron-Ion Collider eRHIC
}

\author{
C. Montag, B. Parker, S. Tepikian, et al \\ Presented at the Particle Accelerator Conference(PAC'05) \\ Knoxville, Tennessee \\ May 16-20, 2005
}

\author{
Collider-Accelerator Department \\ Brookhaven National Laboratory \\ P.O. Box 5000 \\ Upton, NY 11973-5000 \\ www.bnl.gov \\ Managed by \\ Brookhaven Science Associates, LLC \\ for the United States Department of Energy under \\ Contract No. DE-AC02-98CH10886
}

This is a preprint of a paper intended for publication in a journal or proceedings. Since changes may be made before publication, this preprint is made available with the understanding that itwill not be cited or reproduced without the permission of the author. 


\section{DISCLAIMER}

This report was prepared as an account of work sponsored by an agency of the United States Government. Neither the United States Government nor any agency thereof, nor any of their employees, nor any of their contractors, subcontractors, or their employees, makes any warranty, express or implied, or assumes any legal liability or responsibility for the accuracy, completeness, or any third party's use or the results of such use of any information, apparatus, product, or process disclosed, or represents that its use would not infringe privately owned rights. Reference herein to any specific commercial product, process, or service by trade name, trademark, manufacturer, or otherwise, does not necessarily constitute or imply its endorsement, recommendation, or favoring by the United States Government or any agency thereof or its contractors or subcontractors. The views and opinions of authors expressed herein do not necessarily state or reflect those of the United States Government or any agency thereof.

FOR UNCLASSIFIED, UNLIMITED STI PRODUCTS

Available electronically at:

OSTL:

\section{http://www.osti.gov/bridge}

Available for a processing fee to U.S. Department of Energy and its contractors, in paper from:

US. Department of Energy

Office of Scientific and Technical Information

P.O. Box 62

Oak Ridge, TN 37831

Phone: (865) 576-8401

Facsimile: (865) 576-5728

E-mail: reports@adonis.osti.gov

National Technical Information Service (NTIS):

Available for sale to the public from:

U.S. Department of Commerce

National Technical Information Service

5285 Port Royal Road

Springfield, VA 22131

Phone: (800) 553-6847

Facsimile: (703) 605-6900

Online ordering: http://www.ntis.gov/ordering.htm

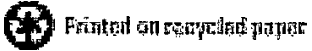




\title{
INTERACTION REGION DESIGN FOR THE ELECTRON-ION COLLIDER ERHIC *
}

\author{
Christoph Montag, B. Parker, S.Tepikian, Brookhaven National Laboratory, Upton, NY 11973, USA \\ D. Wmg, MIT-Bates, Middleton, MA 01949, USA
}

\begin{abstract}
To facilitate the study of collisions between $10 \mathrm{GeV}$ polarized electrons and $100 \mathrm{GeV} / \mathrm{u}$ heavy ions or $250 \mathrm{GeV}$ polarized protons at luminosities in the $10^{33} \mathrm{~cm}^{-2} \mathrm{sec}^{-1}$ range (e-p case), adding a $10 \mathrm{GeV}$ electron storage ring to the existing RHIC complex has been proposed. The interactionregion of this electron-ioncollidereRHIC has to provide the required low-beta focussing, while simultaneously accomodating the synchrotron radiation fan generated by beam separation close to the interaction point, which is particularly challenging. The latest design status of the $\mathrm{R}$ RHIC interaction region will be presented.
\end{abstract}

\section{INTRODUCTION}

The interaction region of an electron-ion collider is particularly challenging. Not only does it have to focus both beams to small, equal spot sizes at the interaction point (IF) to maximize luminosity, but it also has to merge and subsequently separate the two beams which travel in two separate storage rings. This merge and separationis realized by bending the lower-energy electron beam away from the ion beam, which results in a fair amount of synchrotronradiation generated in the interaction region. This radiation ahs to be passed safely through the detector, and an arrangement of masks has to protect the detector from backscattered photons.

In contrast to an earlier design [1], the scheme presented here provides a machine-element free region of $\mathrm{f} 3 \mathrm{~m}$ around the $\mathbb{P}$, at the expense of lower luminosity. This new scheme provides a greater detector acceptance and may therefore be preferred [2].

\section{BEAM SEPARATION}

Generation of synchrotronradiation in the interaction region can be avoided altogether by introduction of a crossing angle, thus providing the required separation of the two beam without the need of magnetic dipole fields. Assuming a minimum required separation of the two beams of $\mathrm{d}=25 \mathrm{~mm}$ at the entrance of the first ion septum quadrupole, and a distance of that magnet from the IP of $l^{*}=5$, the necessary crossing angle can be estimated at $\Theta=5 \mathrm{mrad}$. Introduction of a $5 \mathrm{mrad}$ crossing angle, however, reduces the luminosity by a factor of five due to the relatively long ion bunch length of $\sigma_{z} \approx 20 \mathrm{~cm}$.

\footnotetext{
${ }^{*}$ Work performed under the auspices of the U.S. Department of Energy
}

This can in principle be overcome by introducing a crabcrossing scheme [3, 4], which would roate the long ion bunches into the direction if the oncoming electron bunches, thus resulting in head-on collisions in the comoving frame. The required transverse deflecting voltage of the RF crab cavities is calculated as [4]

$$
V_{\perp}=\frac{c E \tan \theta}{e \omega_{\mathrm{RF}} \sqrt{\beta^{*} \beta_{\mathrm{crab}}}}
$$

where $c$ is the velocity of light, $E$ the beam energy, e the beam particle charge, and $\omega_{\mathrm{RF}}$ the RF voltage. $\beta^{*}$ and $\beta_{\text {crab }}$ denote the $\beta$-functions at the IP and the location of the crab cavity, respectively.

For a $250 \mathrm{GeV}$ proton beam, an RF frequency of $\omega_{\mathrm{RF}}=$ $2 \pi \cdot 200 \mathrm{MHz}$, and $\beta$-functions of $\beta^{*}=1.08 \mathrm{~m}$ and $\beta_{\mathrm{crab}}=$ $400 \mathrm{~m}$, the required RF voltage is calculated as

$$
V_{\perp}=14.4 \mathrm{MV} \text {. }
$$

This voltage is about ten times higher than that for the KEKB crab cavities [5], a system which has been developed but has not yet been installed and tested with beam. Therefore it was decided to design the eRHIC interaction region with zero crossing angle.

To provide head-on collisions the two beams have to be separated by magnetic dipole fields. At the entrance of the first on septum quadrupole, $7.2 \mathrm{~m}$ from the $\mathrm{IP}$, this separation $d$ has to be sufficiently large to provide $20 \sigma_{x, e}$ aperture for the electron beam, $12 \sigma_{x, p}$ for the hadron beam, plus space for the $10 \mathrm{~mm}$ thick septumitself, including vacuum chambers. To minimize the required separation at the septum, horizontal beam sizes $\sigma_{x, e}$ and $\sigma_{x, p}$ at that location therefore have to be kept small. In the case of the ion beam, where the emittance is given by the existing RHIC beam, the horizontal beam size is expressed as

$$
\sigma_{x, p}(7.2 \mathrm{~m}) \propto \sqrt{\beta_{x, p}^{*}+\frac{(7.2 \mathrm{~m})^{2}}{\beta_{x, p}^{*}}} \approx \frac{7.2 \mathrm{~m}}{\sqrt{\beta_{x, p}^{*}}},
$$

because the septum quadrupole is the focusing element closest to the IP. This relation imposes a lower limit on the horizontal $\beta$ function $\beta_{x}^{*}$ at the $\mathbb{P}$, and therefore limits the attainable luminosity.

The electron beam size $\sigma_{x, e}$ at the septum can in principle be minimized by reducing the emittance accordingly. However, since beam sizes at the IP have to be matched, this requires larger electron $\beta$ functions at the IP. Increased $\beta$-functions at the $\mathbb{P}$ result in a larger beam-beam tuneshift - and therefore limit luminosity. Table 1 lists the main interaction region parameters chosen in this design. 


\begin{tabular}{lr}
\hline \hline electrons: & 1278 \\
ring circumference[m] & 120 \\
number of bunches & 5319.5 \\
geometric emittancehor//vert. [nm] & 0.3810 .54 \\
$\beta$ functions hor//vert. [m] & $1.0 \cdot 10^{11}$ \\
particles/bunch & $0.027 / 0.08$ \\
beam-beam tune shifthor./vert. & $174011740 / 870$ \\
damping times hor./vert./long. [turns] & \\
\hline ions: & 3834 \\
ring circumference[m] & 360 \\
number of bun ches & $9.5 / 9.5$ \\
geometric emittancehor./vert. [nm] & 2.1610 .54 \\
$\beta$ functionshor/vert. [m] & $1.0 \cdot 10^{11}(\mathrm{p})$ \\
particles/bunch & $1.0 \cdot 10^{9}(\mathrm{Au})$ \\
& $0.007 / 0.0035$ \\
beam-beam tune shift hor./vert. & $2.2 \cdot 10^{32}$ \\
\hline luminosity [cm ${ }^{-2} \mathrm{sec}^{-1}$ ] & \\
\hline
\end{tabular}

Table 1:Parameter table.

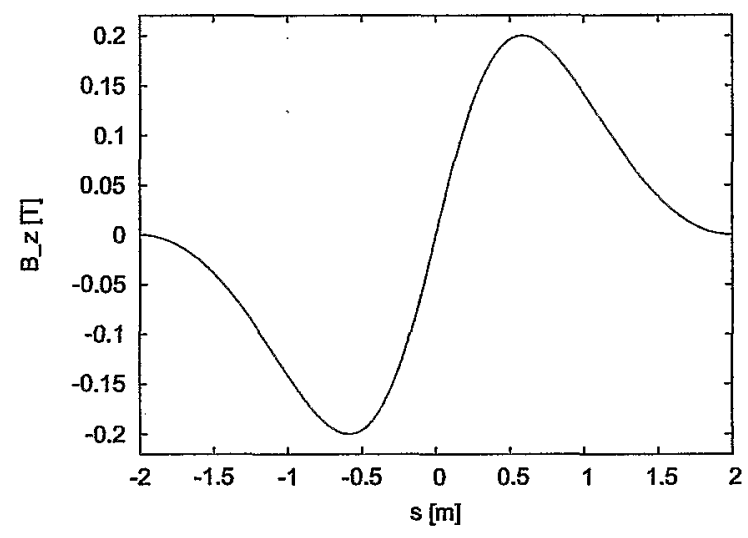

Figure 1: Vertical magnetic field component $B_{z}$ of the detector integrated dipole (DID)used for beam separation. The interaction point $(\mathrm{IP})$ is located at $s=0$.

The required beam separation is provided by dipole coils superimposedon the detector solenoid [6], thus leaving the entire detector volume between the innermostcommon superconducting quadrupoles machine-elementfree. Figure 1 schematically shows the dipole field component of such a detector-integrated dipole (DID) on the solenoid axis.

\section{SYNCHROTRON RADIATION ACCOMODATION}

Photon background in the detector is caused by two types of photons, namely those directly emitted by the beam, and those backscattered from various surfaces in the interaction region. The geometry of the interaction region and the detector has to be chosen such that only synchrotron radiation photons emitted by electrons in the

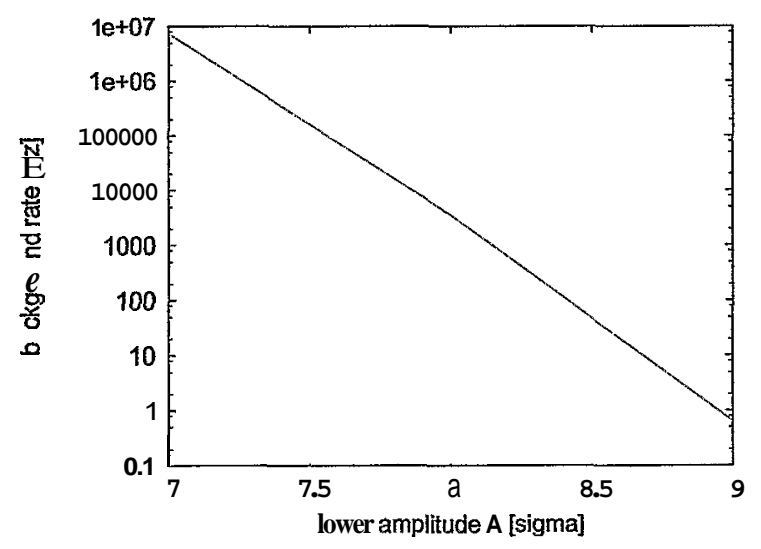

Figure 2: Background rate due to synchrotron radiation photons generated by electrons at amplitudes greater than $\boldsymbol{A}$, as function of $\boldsymbol{A}$.

transverse tails of the distribution can hit surfaces near the detector or the detector itself, while all photons from the core of the beam pass safely through the detector and only hit surfaces at a large distance from the detector. A welldesigned masking system has to ensure that only a small, tolerable fraction of photons backscattered from those surfaces reaches the detector.

The background rate due to direct hits can be estimated by calculating the probability of finding an electron in the transverse beam tails beyond a minimum amplitude $\boldsymbol{A}$ with the bunch crossing rate. With a bunch crossing rate of $28 \mathrm{MHz}$, a background rate of about $1 \mathrm{kFFz}$ is considered sufficiently low. This translates into a minimum aperture requirement for the synchrotronradiation fan such that all photons generatedby electrons at transverse amplitudes of up to $8 \sigma$ pass through the interactionregion, see Figure 2 .

A certain fraction of the synchrotron radiation fan unavoidably hits the septum plate of the first ion septum quadrupole, at $7.2 \mathrm{~m}$ from the IP. This septum is therefore equipped with a dedicated synchrotron radiation absorber to minimize the amount of backscattered photons that may eventually hit the detector, thus contributing to backgound there. Synchrotron radiation masks limit the opening angle of the backscattered radiation fan such that the detector components are shadowed from backscattered photons from the core of the incident fan. Detailed simulation studies are being performed to evaluate and optimize the masking scheme [7]. A top view of the central part of the interaction region is shown in Figure 3.

\section{LOW- $\beta$ FOCUSING}

Focusing of both beams is provided by magnets outside the detector volume, at distances larger then $\mathbf{3}$ from the $\mathbb{P}$. The electron low- $\beta$ triplet is shared by both beams; its effect on the ion beam, however, is practically negligible due to the much higher beam energy. The minimum aper- 


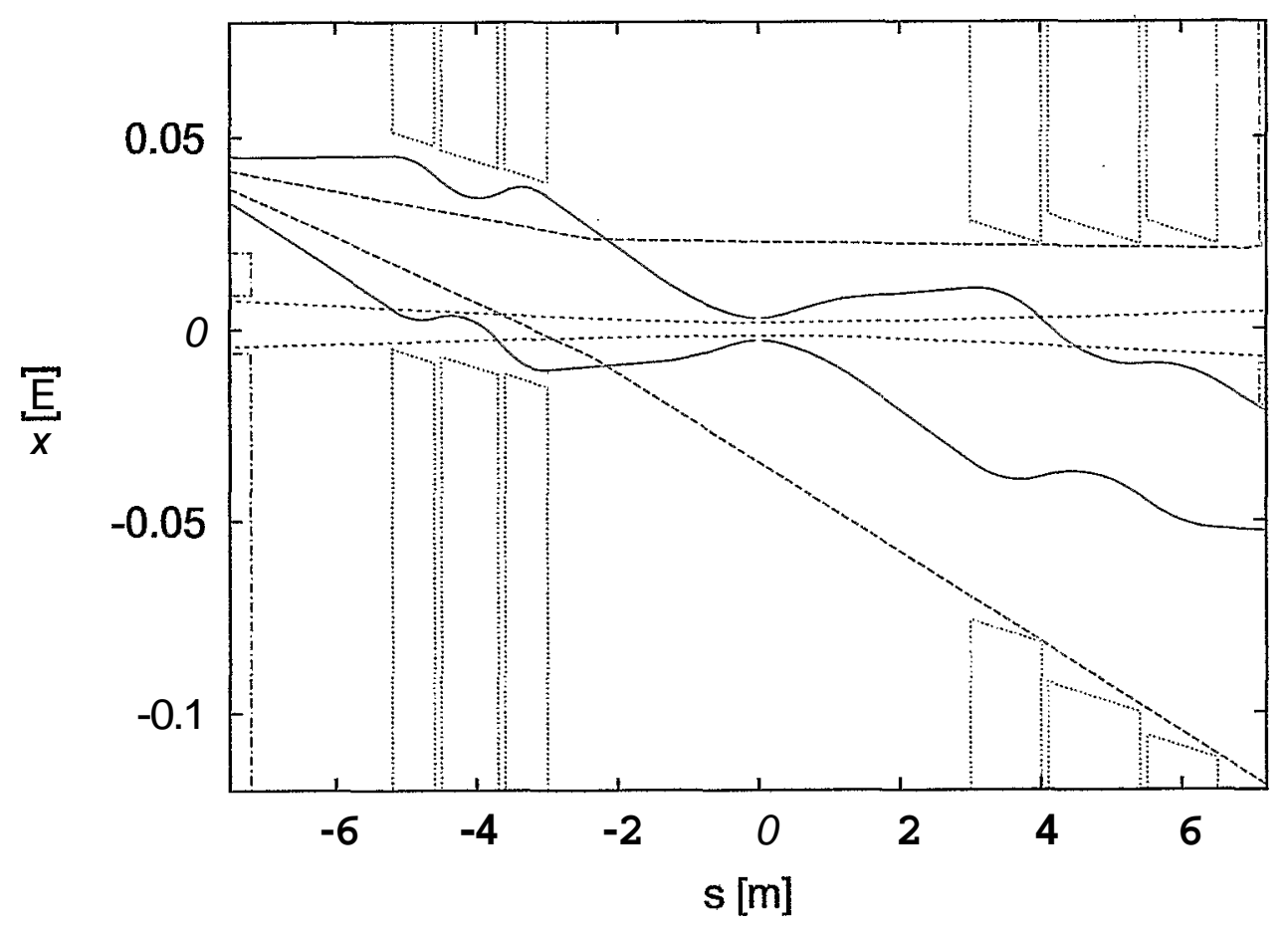

Figure 3: Top view of the eRHIC interaction region with the $20 \sigma$ electron beam (red), the $12 \sigma$ ion beam (blue), and the synchrotronradiation fan generated by the $5 \sigma$ electron beam (green). The apertures of the superconductingelectronlow- $\beta$ quadrupoles (magenta) are tailored according to the sizes of the beams and the fan. The normal-conductingion septum quads are indicated in light blue.

ture of these magnets is determinedby the size of the synchrotron radiation fan from 80 electrons, the 200 , electron beam, and the $12 \sigma_{p}$ ion beam - whichever is largest. On the electron-upstream side this minimum is given by the requirement of sufficient aperture for both beams, as shwon in Figure 3. On the electron-downstream side of the detector, the magnet aperture is dominated by the requirement to safely pass the 80 synchrotron radiation fan. In order to keep peak magnetic fields in the electron downstream quadrupole at the 2 Tesla level, the length of these large-aperturemagnets has been chosen larger than on the electron-upstream side.

On the electron-upstream side, the same magnets could be used; however, it may be more cost efficient to use either normal-conductingmagnets of the same length as downstream, or build a superconductingtriplet of reduced length. The latter would provide a reduced horizontal electron beam size at the upstream septum. This provides an apportunity to reduce the separation angle on the upstream side, which in tum somewhat reduces the synchrotron radiation fan width on the downstream side and therefore the aperture requirementsthere.

Low- $\beta$ focusing of the ion beam is provided by a normalconductingseptum-quadrupoletriplet similar to the one described in Ref. [1], starting at 7.2 $\mathrm{m}$ distance from the IP.

\section{REFERENCES}

[1] C. Montag, B. Parker, S. Tepikian, D. Wang, "Design of an Interaction Region for the Electron-Ion Collider eRHIC", BNL C-A/AP/156 (2004)

[2] B. Surrow, "eRHIC Detector Design Studies - Implications and Constraints on the ep(A) Interaction Region Design", these proceedings

[3] R. B. Palmer, "Energy Scaling, Crab Crossing, and the Pair Problem", SLAC-PUB4707(1988)

[4] K. Oide, K. Yokoya, "Beam-beam collision scheme for storage ring colliders", Phys. Rev. A 40,315 -316, (1989)

[5] KEKB B-Factory Design Report, KEK Report 95-7 (1995)

[6] B. Parker, A. Seryi, "Compensation of the effects of a detector solenoid on the vertical beam orbit in a linear collider", Phys. Rev. ST - Accel. Beams 8,041001 (2005)

[7] J. Beebe-Wang, C. Montag, D. Rondeau, B. Surrow, A. Deshpande, "Synchrotron Radiation in eRHIC Interaction Region", these proceedings 